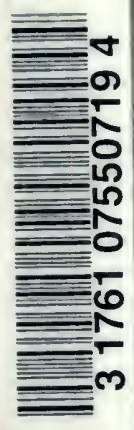

Sils im Engadin, Switzerland. Ordinances, local laws, etc. Uorden forestal per la comunited da Segl 


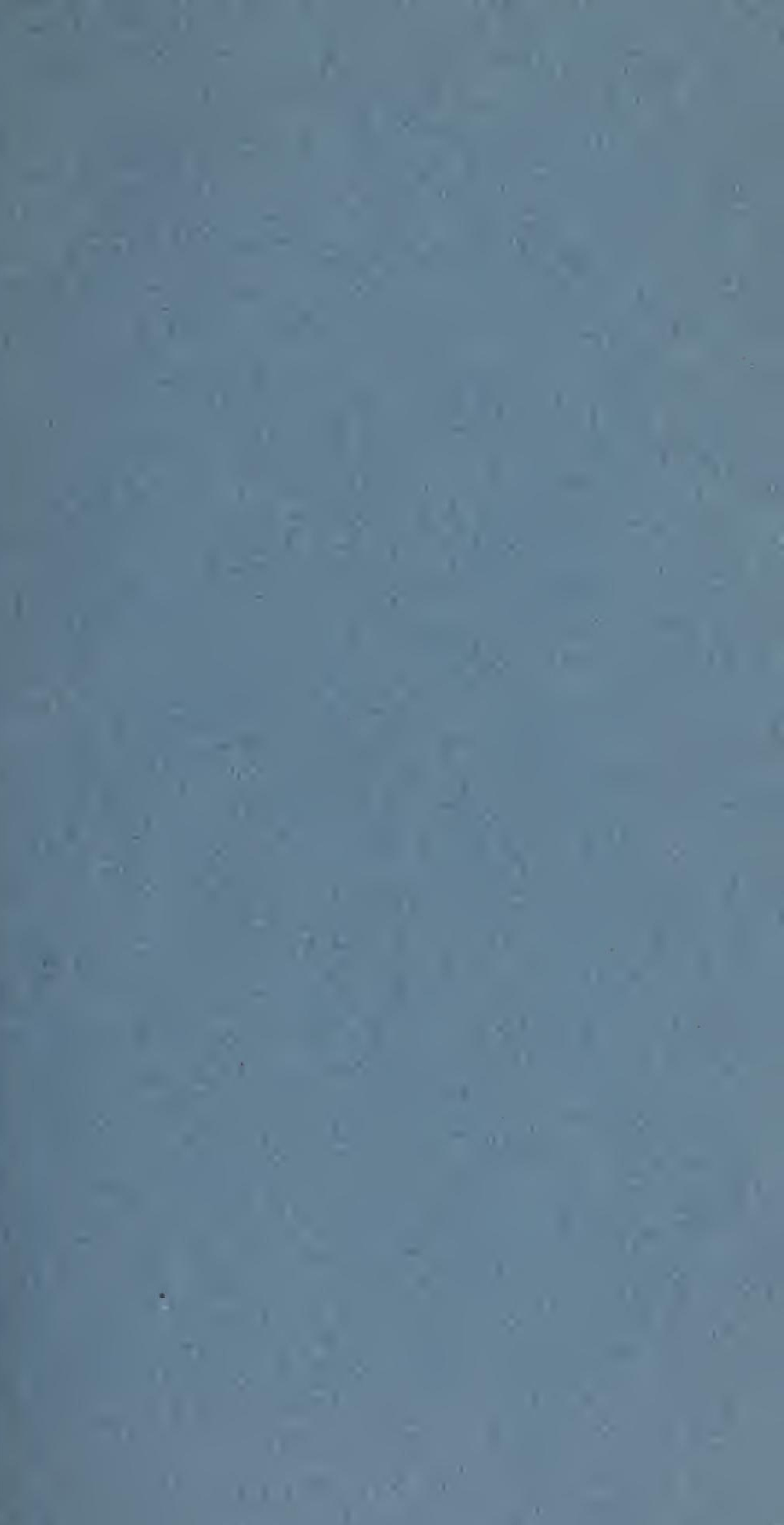




$$
\begin{aligned}
& \text { 象: }
\end{aligned}
$$

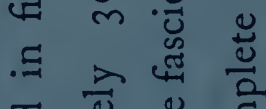

$$
\begin{aligned}
& \text { चु छ } \\
& \text { : } \\
& \text { 己 } \\
& \text { 零总 }
\end{aligned}
$$

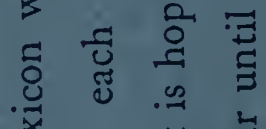

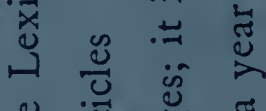

$$
\begin{aligned}
& \text { 胥 }
\end{aligned}
$$




\section{Uorden forestel}

per

\section{la comunited da Segl.}

Touts gods in nossa comunited, taunt comunels sco privats, staun suot survagliaunza superiura federela e chantunela.

La classificaziun dels gods comunels ais regleda tres „piano economic forestel “, in basi del quèl els vegnan giodieus e cultivus.

Lareal (spazi) da noss gods nun po gnir disminuieu infra ils confins fixos tres l'imsüraziun. Cossessas da gods, chi tres evenimaints natürels u tres zercledas gnissan snüdedas, dajan gnir rimplazzedas sainza retard tres implantaziuns giuvnas.

\section{Administrazinn.}

Art. 1.

L'administraziun e survagliaunza da noss gods ais affideda alla commissiun forestela locala, la quela ais rappreschanteda tres il cussagl administrativ comunel e vain in conseguenza eletta per la düreda da duos ans. 
Contabilited e cassa in tuots affer's forestels vegnan mnos tuottaffat separos dalla solita gestiun comunela. Ogni an in occasiun del rendaquint generel dess la commissiun forestela relater detagliedamaing davart sieu operat del an scadien.

\section{Silvicultur.}

Art. 6 .

Il silvicultur' comunel, il quêl daj'esser in possess d'ïna respettiva patenta chantunela, vain elet dalla radunanza comunela per la düreda da trais ans. Sieu salari vain fixo tres contract. Da confirmer il silvicultur in carica, competa alla commissiun forstela.

La serramentaziun da quaist uffiziant sto nella competeuza del Uffizi circuitel.

\section{Art. 7.}

Il silvicultur executescha tuots uordens arvschieus dautoriteds superiuras, sco eir quels della commissiun locala.

El survaglia la polizia forestela e dirigia tuottas lavuors a prô da noss gods. Dal inter andamaint forestel teg'n' el exacta controlla. sco eir da tuottas intredgias e sortidas e funcziunescha conseguentamaing sco „ActuarCassier" per quaist ram dell'administraziun comunela. La contabilited in affers forestels daja gnir preschanteda ogni an als sindicatuors da quints, per la revaisa.

\section{Art. 8.}

Nel cuors del mais d'Avrigl d'ogni an preschaint'el alla commissiun forestela ün piano, accompagno fattibel- 
maing d'ün preventiv per las lavuors da silvicultura. chi avessan da gnir effettuedas in quel an.

Art. 9.

Al silvicultur comunel ais interdit d'as parteciper in qualunque möd saja vi a vendita u negozi da laina.

\section{Gods privats.}

Art. 10.

In tuots gods privats nun po gnir taglio laina, ne per egen adöver ne per esser rendida, sainz' avviser previamaing la commissiun folestela da quetaunt.

Las plauntas, chi paun gnir tagliedas, vegnan signedas. in concordanza cun il silvicultur circuitel, dal silvicultur local.

Per vender laina onr da gods privats ais in pü necessari, per quantums fiu a $60 \mathrm{Fm}$, lacconsentimaint del silvicultur circuitel, per quantiteds pü grandas, quel dell" ota regenza chantunela.

\section{Art. 11.}

Eir l'areal dels gods privats nun po gnir disminuieu. Plazzas snüdedas tres tagls da laina dajan gnir occupedas danövmaing cun plauntas giuvnas. Sün requisiziun pann per tel scopo gnir retrattas dalla vschinauncha las plauntinas adattedas a predsch convenabel. Eir quaistas colturils staun suot survagliamma del silvicultur commel.

\section{Art. 12.}

Cura gniss admiss il tagl da laina in lös cun prievel da lavinas, dessan las tschüchas, las yuclas dajan sainz: excepziun rester nel god, avair in otezaa dad almain $1 \mathrm{~m} 20 \mathrm{~cm}$ sur terra. 
Art. 13.

Las surindichedas disposiziuns in merit als gods privats as baseschan sün la ledscha federela supra ils gods e sün l'uorden forestel chantunel.

\section{Usufrüit prinzipel.}

Art. 14.

La giodia annuela da noss gods dess saimper gnir regleda nels limits fixos tres il respettiv piano d'economia forestel.

Tuotta laina, taunt quella dad arder sco quella da fabrica, dess gnir taglieda, sortida e prepareda exclusivmaing sün quint della vschinauncha e suot controlla e survagliaunza del silvicultur comunel.

Laina da fabrica a predsch redot (laina da zember) vain solum signeda, scha'l quantum sortieu our dal tagl regoler non bastess per currir il bsögn, chi resulta dallas dumandas inoltredas.

Tuotta laina taglieda in noss gods dess gnir sainz' excepziun imsüreda.

\section{Concessiun da laina a predsch redot.}

\section{A. Laina da zember.}

\section{Art. 15.}

Ogni possident dad edifizis in nossa comunited, exceptno fabricats industriels, chi as chatta necessito da reparer tels, po dumander laina da zember our da noss gods comunels vers las seguaintas prescripziuns:

1. Sia petiziun daj'esser addressed'in scrit alla commissiun forestela, in precisand il bsögn ed adöver, ch`el and voul fer. Telas dumandas 
dajan gnir inoltredas, resalro cas urgents " imprevis, in medem temp sco las dumandas da laina dad arder, cioè nel cours del mais Avriyl.

2. La commissiun da se our, u consultand persuma tecnica competenta, examinescha, scha la dumanda saja fundeda e la previsa reparatura da necessitel. Ella decida supra la qualited e quantited della laina.

3. Sün uorden della commissiun in basi della decisiun sarơ la laina signeda, schmersa e pruneda suot survagliaunza del silvicultur in lö accessibel cuı il char.

4. Tuotta laina concessa daja gnil missa in adörel" confuorm a dumanda e concessiun nel spazi dels luos ans subseguaints, partind dalla datta da concessiun.

Da tuottas concessiuns da laina da zember dess la commissim tegner cxacta controlla.

\section{B. Laina dad arder.}

Alt. 16.

Ad ogni famiglia con egna economia, acquia domicilieda, e chi peja tuottas impostas comunelas, vain concess laina dad arder tenor bsögn in fuorma da bïs-chas.

Il quantum dumando less saimper servir per ïs dell' economia sco tela e nun po gnir impondien per scopo d'industria e speculaziun.

Ad economias, chi ham magazin da laina dad ardel derivanta da noss gods, nun rain concess ïngüna laina, lintaunt cha tela surpassa la quantited necessaria per avair laina seclı in avaumz.

Dumandas per laiua dad arder dajan gnir inoltredas 
in scrit al president della commissiun forestela infra il mais Avrigl. Spiro quaist termin, nun saron telas pü consideredas per quel an, cun excepziun da cas imprevis.

La classificaziun dellas büs-chas ais la seguainta e lessan las dumandas gnir inoltredas successivamaing:

I. Büs-cha da $4 \mathrm{~m}^{3}$ laina da tschep dad 1 u $2 \mathrm{~m}$ lungezza.

II. Üna chargia ramma u tschims.

III. Büs-cha da $2 \mathrm{~m}^{3}$ tschüchas regolermaing prunedas.

IV. Büs-cha da $3 \mathrm{~m}^{3}$ tagliöls da 1 u 2 ml lungezza.

V. Eventuel üna chargia föglia (agna), oppüı üna chargia faschinas.

In ans, inua il quantum tschüchas u ramma disponibla non bastess per cuvrir il bsögn, sarò provedieu tres la commissiun in otra maniera. Il predsch per tela laina daja esser unifuorm a quel della büs-cha, ch'ella rimplazza.

\section{Art. 17.}

A predsch redot vain ulteriurmaing concess laina dad arder:

1. Allas alps da Fex e Prasüra, la laina necessaria.

2. Alla societed da chascharia a Maria $9 \mathrm{ml}^{3}$.

3. Alla comunited ecclesiastica evangelica da Segl per l'economia del reverendo $8 \mathrm{~m}^{3}$.

Quaista laina vain pruneda e deda giò in lös, inua ais possibel da transir con mnadüra e char. Per las alps dess la laina dad arder gnir prepareda, scha fattibel, nella prossimited da telas.

\section{Laina gratuita.}

Art. 18.

Laina dad arder gratuita vain concess solum per la chesa comunela e scoula e per l'economia del magister, ils quantums necessaris. 
Süditta laina vain condotta e taglieda sï a quint della vschinauncha.

\section{Ait. 19.}

Per currir tets nours u per reparaturas deventuels tets già existents, cuverts cun niateria inllammabla, nuı conceda la vschinauncha üngüna laina.

Eguelmaing nun vain concess laina our da noss gods per condots d'ova, ne per arder chütschina, chiss ete.

Per pösts e lattas per reparaturas da saivs po gnir concess laina adatteda, premiss cha las dumandas vegnan fattas a temp iitil.

\section{Taxas.}

Art. 20.

Laina dad arder in büs-chas: tagliöls frs. 5.50 per Rm Laina dad arder in büs-chas: tschüchas "2.50 " " Laina dad arder: ramma e tschims "2.- " chargia Laina dad arder: föglia u faschinas "2.- " " Büs-chas per las alps e chascharias " $5.50, \mathrm{Rm}$ Büs-chas per la prevenda

" 5.50 " $"$

Laina da zember peja il $50 \%$ della valur da laina da commerzi.

Per laina da zember pü ordinaria, sco expl. lattas e. pösts per reparaturas da saivs etc., fixa la commissiu forestela da cas in cas il predsch.

Ad eguelas taxas sco sopra sun suottaposts eir quels da Grevasalvas per lur laina dad arder e da zembes, ch'els retiran dalla vschinauncha.

Cun excepziun della laina per las alps, chascharia e prevenda peja il domicilio üna surtaxa per l'usufrüt da $50 \%$ in maximum dellas surindichedas taxas. 
A tuottas quaistas taxas dajan sajmper gnir aggiuntas eir las spaisas da preparaziun e dajan telas gnir scompartidas per ogni categoria da laina u büs-chas separedamaing.

Südittas taxas sun eguelmaing contgnidas nella ledscha d'imposta comunela.

\section{Ulteriuras disposiziuns.}

Art. 21.

Ad accord cum il silvicultur comunel fixa la commissiun forestela di ed ura, inua tuottas büs-chas dajan gnir trattas. Scodün, chi nun retira sieus bigliets da büs-chas sïl di fixo nella bannida, crouda in multa e perda zieva 8 dis il dret per quel an da trer büs-chas. Excepziuns saron reguardedas solum in cas bain motivos.

Art. 22.

L'import taunt per laina dad arder, sco per laina d'inchaunt daja gnir pajo in retrand ils bigliets cun ils rispettivs numers. In ogni cas nun vegnan creditos tels imports per pü lönclı e be in cas excepziunels fiı al 1 December.

Ils ricavos da laina da commerzi e laina da zember derivants da noss gods comunels vegnan remiss, zieva avair redot las spaisas regoleras dell' administraziun forestela e per taunt cha il stedi finanziel della vschinauncha permetta, al fondo forestel (deposit forestel alla banca chantunela).

Ütiliteds derivantas da laina dad arder percunter raun sainz' excepziun in cassa comunela.

Art. 23.

Tuotta laina prepareda in noss gods, saja in büs-chas II otra laina da privats, daja gnir alveda davent e condotta 
our dal god fin al 1 December. Laina da privats, chi resta püi lönch allò, crouda alla rschinammelıa e po quella dispuoner da tela.

\section{Ait. 24.}

In lös, inua pel sflüjer noss gods, saja cun bïs-chas o cun laina da fabrica, füss necessari da passer tres pros privats, po quetaunt dvanter piir cun il 1 Settember davent, evitand però eir allura dannagiamaint da bains privats.

Art. 25.

Laina crudeda a terra tres evenimaints natürels mun dess rester sur an nels gods sainz esser cultiveda, sortida e disposta per quint public, saja per laina da fabrica. per bïs-chas u per inchaunts. Laina da commerzi " da zember, taglieda durante il züj dellas plauntas, daja gnir scorzeda sainza retard.

Art. 26.

Laina da gods comunels crudeda tres evenimaints llatürels sün serrain privat $u$ viceversa resta proprieted del hain, inua ais crudeda. (Vair ledscha civila chantunela.)

Art. 27.

Laina derivanta da noss gods commnels num po da privats esser vendida u cedida a terzas persunas, ne in ne our d'vschinauncha, cun excepciun da laina açuistedil siin via dinchaunt 1 cumpreda a predsch da commerzi.

Ad economias, chi cambian domicili e bandunan nossa comunited, po gnir concess dalla commissiun forestela l'exportaziun da laina daul arder cunter bonificaziun della differenza della taxa redotta al preilsch 
corrent. Sün requisiziun piglia la vschinauncha eir inavous la laina steda concessa al predsch sto pajo.

\section{Art. 28.}

Il dret da raccoglier da qualunque sort laina morta in noss gods ais reservo unicamaing alla vschinauncha, la quela fo eseguir quaista lavur e conceda poi tela laina a privats in fuorma da büs-chas o sün via d'inchaunt public.

Ais severamaing scumando a lavuraints soggiornants in nossa comunited, saja cha tels lavuran per privats " per egen quint, da raccoglier ramma " otra laina in noss gods. Per tels metterò la commissiun forestela sün requisiziun la laina occorrenta a disposiziun vers üna taxa equitabla.

A pastuors e lavuraints comunels ais permiss da porter a chesa la laina mnüda necessaria per lur cuschina, però na per fer commerzi cun tela u la furnir per ïs da lur patruns, tier's ils quêls els sun d'alloggio. La ramma dess però eir in tels cas gnir rammasseda per terra e na guir ruotta giò dallas plauntis.

\section{Usufrüt secundari.}

Alt. 29.

Da fer sternüm, erva e rescha in gods comuncls ais da tuots temps scumando. Eguelmaing proibieu ais da raccoglier sem da god u da fer nuschpignas.

Da raccoglier nuschpignas ais reservo il dret solum alla vschinauncha e dess eventuelmaing eseguir tela lavur suot controlla u del silvicultur' " d'ün member della commissiun foresteli.

In ans da bgeras nuschpignas po la commissiun forestela der gio da telas a predsclı convenabel eir a privats in vschinauncha. 
Per semnannas in gods privats vain in tels ans do gio ils muschels a riflettants sainza provischimn.

\section{A.t. 30.}

Frus-cham, sco sun: grusaidas, giops e bruoch, chi regeteschan ourdvart if goul comunel, paun gnir sdrappos in arantayg del pascul dogui temp e sainza pajer taxas per il quantum rammasso. Percunter in gods nun po gnir sdrappo da tel gener laina, oppür be cunter ün permiss speciel della commissiun u del silvicultur.

\section{Art. 31.}

La pasculaziun cun muaglia müuda ais scumandeda directamaing in tuots noss gods, taunt commels sco privats. Chevras e nuorsas dajan gnir chatschedas per ir sün lur pasculs tres lös arierts e mê tres gods cun plauntas giuvnas.

Per muaglia borina ais la pasculaziun permissa cun il possibel schinagiamaint dels gods. In lös percunter. inua sun culturas e giurras implantaziuns da gool, ais scodüna pasculaziun severamaing interditta. Tels lös dajan possibelmaing guir serros giò cunter pasculs " vias mediante saivs adattedas e sufficiaintas.

\section{Art. 32.}

Fer fö nels gods u nella vicinanza (lin a 50 m almain) da tels ais severamaing scumando. A lavuraints del god po gnir do excepziunelmaing il permiss, a scopo da cuschiner, da fer fö in tels, tres il silvicultur u la commissiun forestela, però eir in tels cas solum in lös, inua ais exclus il prievel d'incendier il god.

Taunt pastuors sco lavuraints dajan esser arvertiens previamaing da quaistas disposiziuns. 


\section{T'urba.}

Art. 33.

Per taunt cha la turbera allas tegias da "Vaüglia" permetta, po ogni economia in vschinauncha cun il permiss della commissiun 1 del silvicultur fer chaver u taglier sï 2 chiargias turba per fer secher ed arder. Tela lavur daja gnir effettueda in möd, ch'ïna controlla tres il silvicultur saja possibla.

Per sternüm ais indistintamaing permiss ad ogni privat, chi tegna muaglia, da chaver in süditta turbera 4 chargias terra da turba. In pü per ogni vacha in estim 3 chargias. La preparaziun da quaist sternüm daja gnir eseguida sainza der dan alla turbera.

Taunt per chaver turba, sco eir per fer sternüm nella turbera, daja gnir fatta la resp. dumanda insembel con quella per la laina dad arder.

\section{Contravenziuns e fillas.}

\section{Art. 34 .}

Tuots abitants da nossa comunited, taunt indigens sco foresters, dessan s'unifuormer ed as suottametter allas prescripziuns del preschaint "uorden forestel".

Ogni ün, chi ais comprovo, d'avair contrafat a quel, daja gnir denunzio e punieu sainza risguard alchün, ne da persunas da rango ne uffizi. Que ais il dovair d'ogni abitant da nossa comunited, da notificher immediatanıaing alla commissiun forestela $\mathrm{u}$ al silvicultur tuots cas da contrafacziuns da god ad el contschaints. Sieu nom nun sarò paleso e survain el in pü $1 / 3$ della relativa falla. Occultaziuns da terzas persunas saron punidas tenor il cas. 
Fallas regnan applichedas sco segua:

1. Contrafacziuns dels art. 10,11 e 12 (gods privats) cun firs. 5-50, oppür cun frs. 5-10 per ogni Fm laina taglieda sainza permiss.

๖. Cunter l'art. 15 cifr. 4 cun frs. 5 per ogni Fm, nel cas cha la laina nun füss missa in adöver aunz spiro il termin fixo, e cun frs. 5-20 per Fm, scha la laina füss steda impondida per oter scopo.

3. Per retard da trer las biis-chas (art. 21 ) fr. 1 per di.

4. Contrafacziuns cunter l'art. 27 cun frs. $5-20$.

5. Contrafacziuns dels art. 28 e 29 cun frs. 2-10.

6. Contrafacziuns del art. 30 cun frs. $2-5$.

7. Contrafacziuns del art. 31 cun fr. 1 per cho e per routa. (Da not falla redobleda.)

8. Fer fö nels gods, art. 32, cun frs. 2-100.

9. Contrafacziuns del art. 33 cun frs. 2-10.

10. Taglier plauntinas da fruonzla e dannagiamaint d'implantaziuns e colturas fr's. 5-100.

11. Taglier plauntas pü grandas sechas frs. $5-15$ per Fm.

Taglier da telas verdas cun frs. $10-30$ per Fm ed in pü bonificher la plaina valur della laina taglieda.

Contravenziuns in gods durante la not saron adüna multedas cun il maxim del chastih. Tuotta laina da contravenziun sarò sequestreda e missa a disposiziun della vschinauncha. A privats, chi mainan davent büschas u laina d'inchaunt dad oters, vain inflit üı chastilı da frs. 5 per numer e haun in pü il dovair da rimplazzer la laina all'abitaziun del dannagio. In tels cas sun ils patruns responsabels per lur famagls e vittürins. 
Fallas inflittas dalla commissiun forestela comunela non excludin eventuels chastihs dad instanzas superiuras (2) viceversa.

\section{Disposizinns transitorias.}

Quaist uorden forestel ais baso sün l'uorden forestel clantunel e rimplazza quel del 27 Favrer 1903. El füt sancziuno ed accepto d'üna lod. radunanza comunela suot d. d. 16 e $19 \mathrm{Meg}$ 1909. Scumbod ch' el sarò sancziuno dall'ota regenza chantunela, aintra el in vigur.

Segl, Meg 1909.

In nom del cussagl comunel:

J. Corai, president

G. Garlmann, actuar.

Die vorstehende Waldordnung der Gemeinde Sils i. E. wird anmit vom Kleinen Rate genelnmigt.

Chur, den 6. Juli 1909.

Der Präsident i. V.:

Raschein.

Der Kanzleidirektor:

G. Fient. 

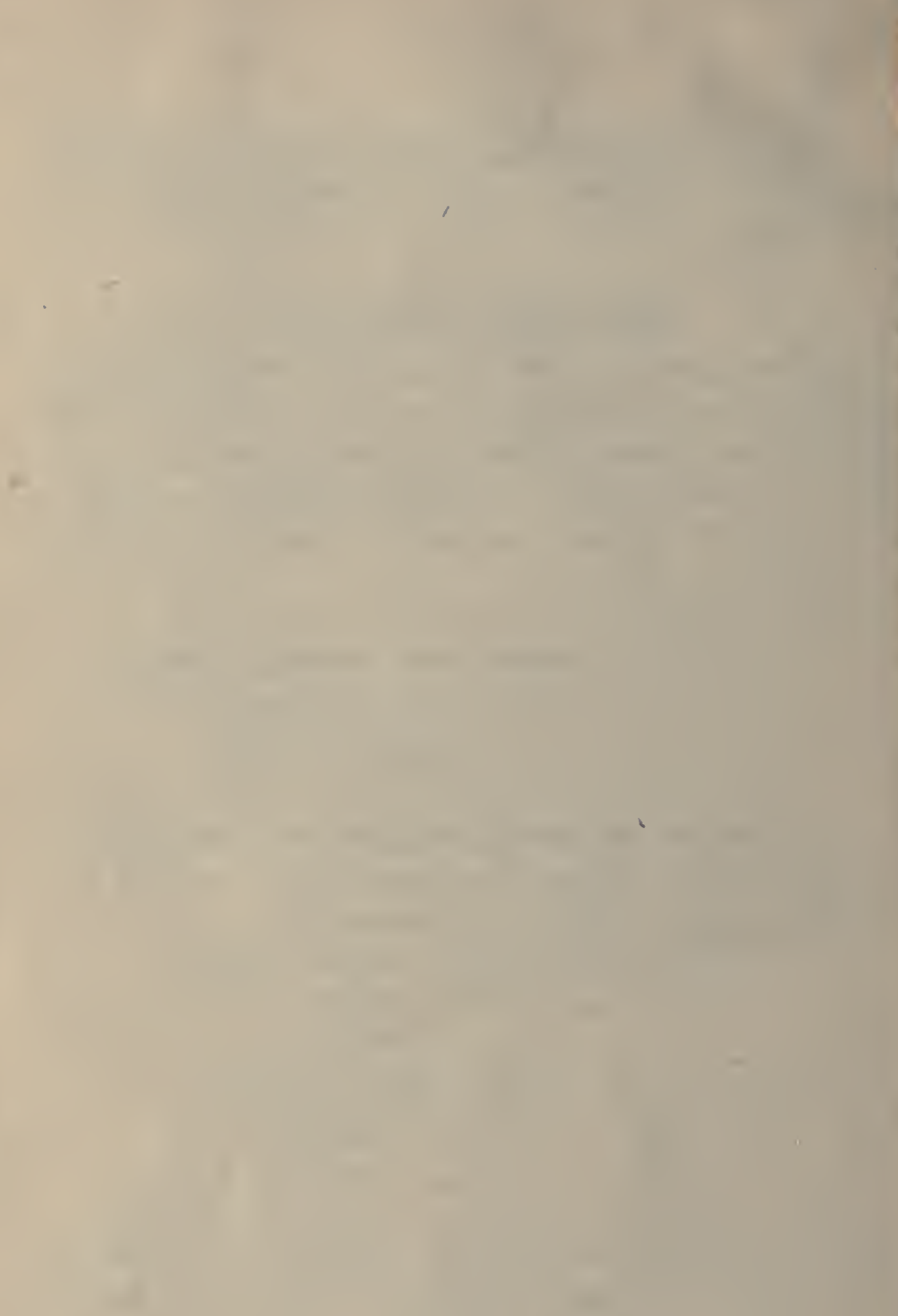


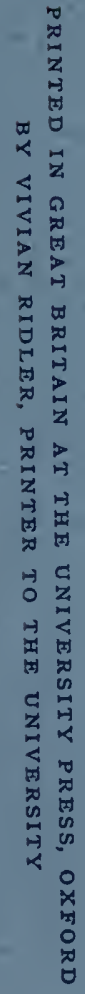



SD

636

$554 \mathrm{Al}_{4} 7$
Sils im Engadin, Switzerland. Ordinances, local laws, etc. Uorden forestal per la comunited da Segl

BioMed

\section{PLEASE DO NOT REMOVE}

CARDS OR SLIPS FROM THIS POCKET

UNIVERSITY OF TORONTO LIBRARY 
